UDC 62-752 (045),

DOI: $10.18372 / 1990-5548.52 .11866$

L. M. Ryzhkov

\title{
CONTROL SYSTEM SYNTHESIS OF INDICATOR DYNAMIC VIBRATION ABSORBERS
}

Aircraft and Space Systems Department National Technical University of Ukraine "Ihor Sikorsky Kyiv

Polytechnic Institute," Kyiv, Ukraine

E-mail: lev_ryzhkov@rambler.ru

\begin{abstract}
Dynamic vibration absorbers of indicator type are analyzed. In such absorbers direct interaction between object and absorber is absent. It is shown that such absorbers are effective even for simple controllers. For analyze the linear matrix inequalities are used. Dynamic vibration absorbers of indicator type are analyzed. Their positive feature is that there is no need to change the design of the main device because of in such absorbers direct interaction between object and absorber is absent. It is shown that using proposed formulas for parameters of controller we can obtain effective absorber with simple controller. For analyze the linear matrix inequalities are also used. The problem is analyzed as $H_{\infty}$-synthesis problem. It is shown that using linear matrix inequalities we can also obtain very effective absorber.
\end{abstract}

Index Terms-Dynamic vibration absorber; controller; linear matrix inequalities.

\section{INTRODUCTION}

Dynamic vibration absorbers (DVA) are an effective means of reducing the impact of external disturbances on the devices. The disadvantage of the classic DVA is direct contact of the object and the absorbers. This requires changing the design of the device, increasing its size. For miniature devices this approach is often technically impossible. In articles [1], [2] are considered the analysis and synthesis of active indicator DVA, where there is no direct interaction between object and absorbers. The scheme of such DVA is shown in Fig. 1.

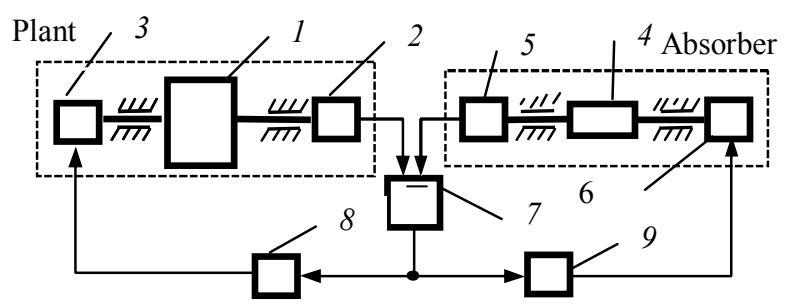

Fig. 1. Indicator dynamic vibration absorber

We assume that the plant has moving mass 1 , angle sensor 2 and torque sensor 3. On the axis parallel to the axis of the plant, set the absorbers, which also has a moving mass 4 , angle sensor 5 and torque sensor 6 . The sensors output signals are compared 7 and submitted by controllers 8,9 to the torque sensors.

In this scheme, there is no mechanical interaction of moving masses, that is, the implementation of the scheme does not require modification of the plant. In such absorbers the moving mass of absorbers is, in fact, the only indicator of the movement of plant mass. This is what explains the name of the absorbers.

\section{PROBLEM STATEMENT}

In classical DVA the moments acting on the moving masses of the plant and the absorber are equal in magnitude and opposite in direction. In the scheme, they can be different. This is ensured by proper choice of the laws of control. Consider the effectiveness of such absorber synthesized by given in articles [1], [2] method, and in the case of theory of linear matrix inequalities (LMN) using [3].

\section{PRoblem Solution}

Consider DVA as a system of automatic control. To Figure 1 correspond equations

$$
\begin{aligned}
& W_{1}(s) \varphi_{1}=M-W_{K 1}(s)\left(\varphi_{1}-\varphi_{2}\right) ; \\
& W_{2}(s) \varphi_{2}=W_{K 2}(s)\left(\varphi_{1}-\varphi_{2}\right),
\end{aligned}
$$

where $\varphi_{1}, \varphi_{2}$ are displacements of the plant and the absorber; $W_{1}(s)=I_{1} s^{2}+c_{0} ; W_{2}(s)=I_{2} s^{2}$ are transfer functions; $I_{1}, I_{2}$ are moments of inertia; $c_{0}$ is the stiffness coefficient; $W_{K 1}(s), W_{K 2}(s)$ are transfer functions of controller.

To classic DVA correspond such transfer functions of controller: $W_{K 1}(s)=W_{K 2}(s)=h s+c$. Therefore assume

$$
\begin{aligned}
& W_{K 1}(s)=h_{1} s+c_{1} ; \\
& W_{K 2}(s)=h_{2} s+c_{2}
\end{aligned}
$$

and write

$$
\begin{aligned}
& \left(I_{1} s^{2}+W_{K 1}+c_{0}\right) \varphi_{1}-W_{K 1} \varphi_{2}=M \\
& \left(I_{2} s^{2}+W_{K 2}\right) \varphi_{2}-W_{K 2} \varphi_{1}=0 .
\end{aligned}
$$


Rewrite these equations in the form

$$
\begin{aligned}
& \left(s^{2}+n_{0}^{2}+2 \xi_{1} n_{1} s+n_{1}^{2}\right) \varphi_{1}-\left(2 \xi_{1} n_{1} s+n_{1}^{2}\right) \varphi_{2}=w, \\
& \left(s^{2}+2 \xi_{2} n_{2} s+n_{2}^{2}\right) \varphi_{2}-\left(2 \xi_{2} n_{2} s+n_{2}^{2}\right) \varphi_{1}=0,
\end{aligned}
$$

where

$$
\begin{aligned}
& w=\frac{1}{I_{1}} M ; n_{0}^{2}=\frac{c_{0}}{I_{1}} ; n_{1}^{2}=\frac{c_{1}}{I_{1}} ; n_{2}^{2}=\frac{c_{2}}{I_{2}} ; \xi_{1}=\frac{h_{1}}{2 I_{1} n_{1}}, \\
& \xi_{2}=\frac{h_{2}}{2 I_{2} n_{2}} .
\end{aligned}
$$

The optimal parameters of DVA are [2]

$$
\begin{gathered}
\mu_{1}=\frac{n_{1}}{n_{0}}=\frac{1}{1+\rho} \sqrt{2 \rho} ; \quad \xi_{1}=\frac{\rho}{2} \sqrt{\frac{3+\rho}{(1+\rho)(2+\rho)}}, \\
\mu_{2}=\frac{n_{2}}{n_{0}}=\frac{1}{1+\rho} \sqrt{\frac{2}{2+\rho}} ; \quad \xi_{2}=\frac{1}{2} \sqrt{\frac{\rho(3+\rho)}{1+\rho}} \\
\delta_{m}=\frac{\rho+2}{\sqrt{\rho(\rho+4)}},
\end{gathered}
$$

where $\rho=\lambda_{2} q=\frac{h_{1}}{h_{2}} \frac{I_{2}}{I_{1}} ; \lambda_{2}=\frac{h_{1}}{h_{2}} ; \delta_{m}$ is the maximal value of relative transfer factor of DVA.

Relative transfer function of DVA is $\delta(s)=\frac{W(s)}{W(0)}$, where $W(s)=\frac{\varphi_{1}(s)}{w(s)}$. We see that optimal parameters are functions only one parameter $\rho$.

In Figure 2 are represented relations $\delta\left(\omega / n_{0}\right)$ for three values of parameter $\rho$. Note, that for classical DVA with optimal parameters for $q=0,1$ we have $\delta_{m k}=4.58$.

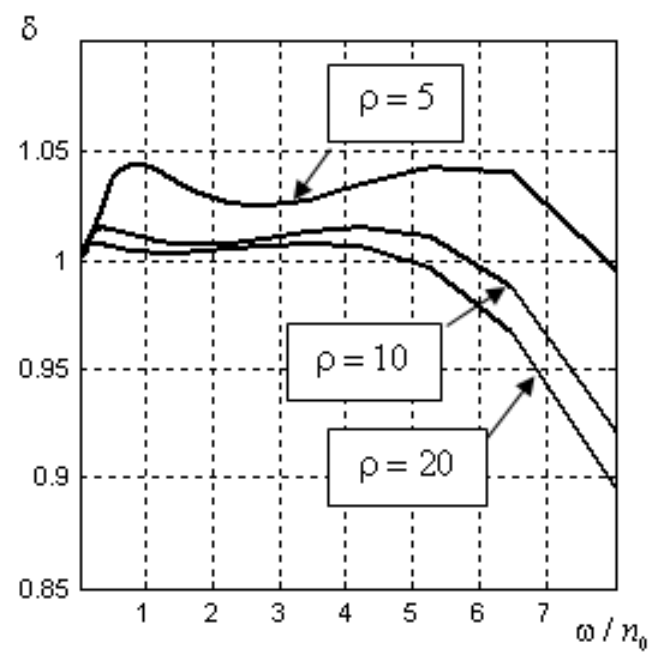

Fig. 2. Relations $\delta\left(\omega / n_{0}\right)$
The effectiveness of DVA is specified not mass ratio $q$, as in classical DVA, but by the parameter $\rho=\lambda_{2} q$. Therefore increasing the parameter $\lambda_{2}$ we can obtain effective DVA even for little mass of absorber.

Now fulfill the controller synthesis using linear matrix inequalities for standard form system (Fig. 3) with equations

$$
K(s):\left\{\begin{array}{l}
\dot{\varsigma}=A_{K} \varsigma+B_{K} y, \\
U=C_{K} \varsigma+D_{K} y .
\end{array}\right.
$$

$$
P(s):\left\{\begin{array}{l}
\dot{X}=A X+B_{1} w+B_{2} U, \\
\mathrm{z}=C_{1} X+D_{11} w+D_{12} U, \\
\mathrm{y}=C_{2} X+D_{21} w+D_{22} U,
\end{array}\right.
$$

We have

$$
\begin{aligned}
& \dot{X}_{C L}=A_{C L} X_{C L}+B_{C L} w \\
& z=C_{C L} X_{C L}+D_{C L} w
\end{aligned}
$$

where

$$
\begin{aligned}
X_{C L} & =\left[\begin{array}{ll}
X & \zeta
\end{array}\right]^{\mathrm{T}}, \\
A_{C L} & =\left[\begin{array}{cc}
A+B_{2} D_{K} C_{2} & B_{2} C_{K} \\
B_{K} C_{2} & A_{K}
\end{array}\right], \\
B_{C L} & =\left[\begin{array}{c}
B_{1}+B_{2} D_{K} D_{21} \\
B_{K} D_{21}
\end{array}\right], \\
C_{C L} & =\left[\begin{array}{ll}
C_{1}+D_{12} D_{K} C_{2} & D_{12} C_{K}
\end{array}\right], \\
D_{C L} & =\left[D_{11}+D_{12} D_{K} D_{21}\right] .
\end{aligned}
$$

Write equations of motion in the form

$$
\begin{aligned}
& \ddot{\varphi}_{1}+n_{0}^{2} \varphi_{1}=w+u_{1} ; \\
& \ddot{\varphi}_{2}=-\frac{1}{q} u_{2} .
\end{aligned}
$$

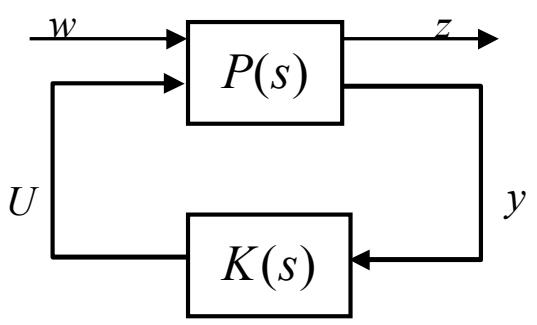

Fig. 3. Standard form of closed-loop system

Specify new variables $x_{1}=\varphi_{1} ; x_{2}=\varphi_{2} ; x_{3}=\dot{\varphi}_{1}$; $x_{4}=\dot{\varphi}_{2}$. As control signal assume $y=\varphi_{1}-\varphi_{2}$ and as output signal assume $z=\varphi_{1}$. 
In standard form we can write

$$
\begin{aligned}
& \dot{X}=A X+B_{1} w+B_{2} u, \\
& z=C_{1} X, \\
& y=C_{2} X,
\end{aligned}
$$

where

$$
\begin{gathered}
X=\left[x_{1}, x_{2}, x_{3}, x_{4}\right]^{\mathrm{T}}, \\
x_{3}=\dot{x}_{1} ; x_{4}=\dot{x}_{2},
\end{gathered}
$$

$$
\begin{aligned}
& A=\left[\begin{array}{cccc}
0 & 0 & 1 & 0 \\
0 & 0 & 0 & 1 \\
-n_{0}^{2} & 0 & 0 & 0 \\
0 & 0 & 0 & 0
\end{array}\right], B_{1}=\left[\begin{array}{l}
0 \\
0 \\
1 \\
0
\end{array}\right], B_{2}=\left[\begin{array}{ll}
B_{21} & B_{22}
\end{array}\right], \\
& B_{21}=\left[\begin{array}{llll}
0 & 0 & -1 & 0
\end{array}\right]^{\mathrm{T}}, B_{22}=\left[\begin{array}{llll}
0 & 0 & 0 & \frac{1}{q}
\end{array}\right]^{\mathrm{T}},
\end{aligned}
$$

$C_{1}=\left[\begin{array}{llll}1 & 0 & 0 & 0\end{array}\right], C_{2}=\left[\begin{array}{llll}1 & -1 & 0 & 0\end{array}\right], U=\left[\begin{array}{l}u_{1} \\ u_{2}\end{array}\right]=K y$.

In our case $D_{11}=0 ; D_{12}=0 ; D_{21}=0 ; \quad D_{22}=0 ;$ $D_{K}=0$. Therefore

$$
\begin{aligned}
A_{C L} & =\left[\begin{array}{cc}
A & B_{2} C_{K} \\
B_{K} C_{2} & A_{K}
\end{array}\right], \quad B_{C L}=\left[\begin{array}{c}
B_{1} \\
0
\end{array}\right], \\
C_{C L} & =C_{1}, \quad D_{C L}=0 .
\end{aligned}
$$

Assume this problem as $H_{\infty}$-synthesis problem. The H- $\infty$ norm of transfer function $\frac{x_{1}(s)}{w(s)}$ will be less than number $\gamma$ if and only if there exist a symmetric matrix $Q$ such that the following LMIs are satisfied

$$
\begin{gathered}
{\left[\begin{array}{ccc}
A_{C L} Q+Q A_{C L}^{\mathrm{T}} & B_{C L} & Q C_{1}^{\mathrm{T}} \\
B_{C L}^{\mathrm{T}} & -I & 0 \\
C_{1} Q & 0 & -\gamma^{2} I
\end{array}\right]<0,} \\
Q=Q^{\mathrm{T}}>0 .
\end{gathered}
$$

For analysis will use Matlab function "hinflmi". To obtain the minimal value of parameter $\gamma$ ifs value was not preliminary specified. For calculations was assumed $q=0.1 ; \quad n_{0}=10 \mathrm{~s}^{-1}$. The equations of controller were found and after this the transfer functions of controller and the DVA relative transfer function were found.

Using transfer functions of controller $W_{K 1}(s)$, $W_{K 2}(s)$ we can write the DVA equations

$$
\begin{aligned}
& \left(s^{2}+n_{0}^{2}\right) \varphi_{1}=w-W_{K 1}(s)\left(\varphi_{1}-\varphi_{2}\right), \\
& s^{2} \varphi_{2}=\frac{1}{q} W_{K 2}(s)\left(\varphi_{1}-\varphi_{2}\right),
\end{aligned}
$$

Dynamic vibration absorbers transfer function

$$
W(s) \frac{s^{2}+\frac{1}{q} W_{K 2}(s)}{s^{4}+\left[n_{0}^{2}+W_{K 1}(s)+\frac{1}{q} W_{K 2}(s)\right] s^{2}+n_{0}^{2} \frac{1}{q} W_{K 2}(s)}
$$

and relative transfer function.

As result were obtained such transfer functions of controller

$$
\begin{aligned}
& W_{k 1}(s)=\frac{-1,983 \cdot 10^{7} s^{3}-3,443 \cdot 10^{11} s^{2}+1,82 \cdot 10^{12} s+1,209 \cdot 10^{12}}{s^{4}+1,783 \cdot 10^{4} s^{3}+3,328 \cdot 10^{6} s^{2}+4,998 \cdot 10^{8} s+3,904 \cdot 10^{11}}, \\
& W_{k 2}(s)=\frac{1,041 \cdot 10^{6} s^{3}-6,83 \cdot 10^{7} s^{2}+2,37 \cdot 10^{10} s+3,106 \cdot 10^{8}}{s^{4}+1,783 \cdot 10^{4} s^{3}+3,328 \cdot 10^{6} s^{2}+4,998 \cdot 10^{8} s+3,904 \cdot 10^{11}} .
\end{aligned}
$$

In Figure 4 the relations $\delta\left(\omega / n_{0}\right)$ when LMI theory was used (line 1 ) and optimal parameters (4) for $\rho=50$ were used (line 2) are shown.

We see that DVA of indicator type is effective even when using simple controller. This is because in this DVA control signals acting on the object and the moving mass of absorber are different.

\section{CONCLUSION}

Dynamic vibration absorbers of indicator type are an effective means of reducing the impact of external disturbances on the devices. Their positive feature is that there is no need to change the design of the main device. Such DVA are very effective even for simple controllers.

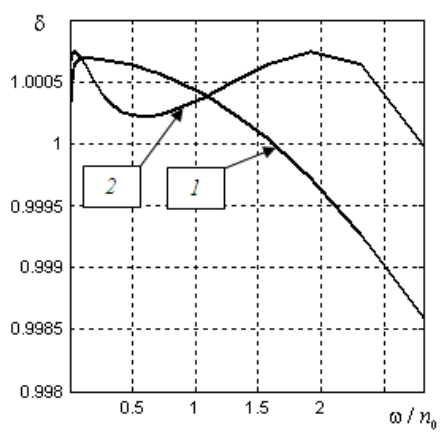

Fig. 4. Relations $\delta\left(\omega / n_{0}\right)$ 


\section{REFERENCES}

[1] L. M. Ryzhkov and V. M. Simonenko. "About the synthesis of dynamic faders of oscillations," Scientific news of NTUU "KPI," no. 2, pp. 92-94, 2000. (in Ukrainian)

[2] L. M. Ryzhkov. "Optimization of parameters of electromechanical dynamic faders of oscillations," Scientific news of NTUU "KPI," no. 1, pp. 57-61, 2003. (in Ukrainian)
[3] D. V. Balandin and I. A. Fedotov. "Synthesis of an active dynamic vibration damper using linear matrix inequalities," Mathematical modeling and optimal control. Bulletin of the Nizhny Novgorod University. N. I. Lobachevsky, no. 6, pp. 153-159, 2007. (in Russian)

Received January 10, 2017

Ryzhkov Lev. Doctor of engineering. Professor.

Aircraft and Space Systems Department National Technical University of Ukraine "Ihor Sikorsky Kyiv Polytechnic Institute", Kyiv, Ukraine.

Education: Kyiv Politechnic Institute, Kyiv, Ukraine. (1971)

Research interests: navigation devices and systems.

Publications: 247.

E-mail: lev_ryzhkov@rambler.ru

\section{Л. М. Рижков. Синтез системи керування індикаторних динамічних гасників коливань}

Проаналізовано динамічні гасники коливань індикаторного типу. Перевага таких гасників полягає у відсутності прямої взаємодії між об'єктом та гасником. Показано, що використовуючи запропоновані формули для параметрів контролера можна отримати ефективний гасник з простим контролером. Для аналізу використовуються також лінійні матричні нерівності. Задачу проаналізовано як задачу $H_{\infty}$-синтезу. Показано, що використовуючи лінійні матричні нерівності також можна отримати дуже ефективні гасники.

Ключові слова: динамічний гасник коливань; регулятор; лінійні матричні нерівності.

Рижков Лев Михайлович. Доктор технічних наук. Професор.

Кафедра приладів та систем керування літальними апаратами, Національний технічний університет України «Київський політехнічний інститут ім. Ігоря Сікорського», Київ, Україна.

Освіта: Київський політехнічний інститут, Київ, Україна, (1971).

Напрям наукової діяльності: навігаційні прилади та системи.

Кількість публікацій: 247.

E-mail: lev_ryzhkov@rambler.ru

\section{Л. М. Рыжков. Синтез системы управления индикаторных динамических гасителей колебаний}

Проанализированы динамические гасители колебаний индикаторного типа. Преимущество таких гасителей заключается в отсутствии прямого взаимодействия между объектом и гасителем. Показано, что используя предложенные формулы для параметров контроллера можно получить эффективный гаситель с простым контроллером. Для анализа используются также линейные матричные неравенства. Задача проанализирована как задача $H_{\infty}$-синтеза. Показано, что используя линейные матричные неравенства можно получить очень эффективные гасители.

Ключевые слова: динамический гаситель колебаний; регулятор; линейные матричные неравенства.

Рыжков Лев Михайлович. Доктор технических наук. Профессор.

Кафедра приборов и систем управления летательными аппаратами, Национальный технический университет Украины «Киевский политехнический институт им. Игоря Сикорского», Киев, Украина.

Образование: Киевский политехнический институт, Киев, Украина (1971).

Направление научной деятельности: навигационные приборы и системы.

Количество публикаций: 247.

E-mail: lev_ryzhkov@rambler.ru 